\title{
Statistical Control Chart and Neural Network Classification for Improving Human Fall Detection
}

\author{
Fouzi Harrou $^{a}$, Member, IEEE, Nabil Zerrouki ${ }^{b}$ \\ ${ }^{a}$ King Abdullah University of Science and Technology, \\ CEMSE Division, Thuwal, 23955-6900, Saudi Arabia \\ Email: fouzi.harrou@kaust.edu.sa
}

\author{
Ying Sun ${ }^{a}$, Amrane Houacine ${ }^{b}$ \\ ${ }^{b}$ University of Sciences and Technology Houari Boumédienne \\ Algeria, LCPTS, Faculty of Electronics and Computer Science
}

\begin{abstract}
This paper proposes a statistical approach to detect and classify human falls based on both visual data from camera and accelerometric data captured by accelerometer. Specifically, we first use a Shewhart control chart to detect the presence of potential falls by using accelerometric data. Unfortunately, this chart cannot distinguish real falls from fall-like actions, such as lying down. To bypass this difficulty, a neural network classifier is then applied only on the detected cases through visual data. To assess the performance of the proposed method, experiments are conducted on the publicly available fall detection databases: the University of Rzeszow's fall detection (URFD) dataset. Results demonstrate that the detection phase play a key role in reducing the number of sequences used as input into the neural network classifier for classification, significantly reducing computational burden and achieving better accuracy.
\end{abstract}

\section{INTRODUCTION}

Falls are an important health care concern, especially for seniors [1]. As shown in the study [2] given by the World Health Organization, $30 \%$ of population older than 65 years old falls at least once each year. Furthermore, $47 \%$ of those who have fallen can not get up without help [3]. By 2020, falls are predicted to increase medical care expenditures by $\$ 43.8$ billion [4]. There is a clear need for reliable fall detection and classification systems that will improve quality of life and increase levels of safety [5], [6].

Along past the two decades, researchers and engineers have developed several fall detection techniques that generally could be split into two main classes: non-computer vision based approaches and computer vision based approaches [7][9]. Non-computer-vision-based fall detection approaches are usually rested on information captured by sensors. These methods use sound, vibrations, and human body movements to detect a fall [7], [10]. On the other hand, computer-visionbased fall detection methods are relied on information obtained from images and videos [7], [11]. In this paper, we propose a fall detection and classification technique based on both wearable sensors and video monitoring systems. The main goal behind such combination is to achieve low computational costs and short execution time together with suitable fall detection performance.

This paper addresses the problem of detecting fall events within a statistical framework. The problem of detecting human falls is addressed as an anomaly detection problem.
Specifically, the fall detection and classification are based on the accelerometric data and variations in human silhouette shape. We use Shewhart control chart to detect a potential fall by using the accelerometric data. The main advantage of this chart is that it can be easily implemented in real time because of its low computational cost [12]-[14]. However, the Shewhart detection chart cannot differentiate real falls from fall-like events. This confusion is mainly caused by the degree of similarity between the features extracted from wearable sensors of such action. To overcome this confusion, the camera data is exploited. Indeed, we used Shewhart to identify features corresponding to fall events that are useful for fall classification purposes. Only features corresponding to detected falls will be used in classification phase. Once the fall is detected using sensors data, the neural network classifier [15] is applied on the data collected from RGB camera to distinguish between real falls from fall-like events. Such a choice is motivated by the greater flexibility of neural network algorithm to deal with linear and nonlinear data. In this study, we apply this strategy to the publicly available fall detection databases from the university of Rzeszow's.

The rest of the paper is organized as follows: Section II presents fall detection based on accelerometric data and outlines the Shewhart monitoring chart and its use in fall detection. Section III presents the vision-based fall classification steps and the neural network classifier used to distinguish real falls from fall-like events. Section IV evaluates the performance of the proposed method, and Section V concludes this study with some remarks.

\section{Fall Detection Using Accelerometric Data}

Fall detection, which is a binary decision making process, consists in identifying falls from non-falls events based on some relevant data features. In this work, we present fall detection algorithms based on Shewhart chart.

\section{A. Shewhart monitoring chart}

This paper addresses the problem of detecting falls as an anomaly detection problem. The anomaly occurs at the moment of a fall. Detecting the particular anomalies that occurs in a monitored system is based on checking whether the current measurements are statistically different from the a priori known faultless measurements (i.e., measurements without 
8th International Conference on Modelling, Identification and Control (ICMIC-2016)

Algiers, Algeria- November 15-17, 2016

anomalies). Towards this end, Shewhart chart operates directly on the measurements acquired from the process without any transformation of data. The Shewhart chart uses the observed data at the current time point alone for making decisions about the process performance at the current time point. More specifically, Shewhart charts evaluate the performance of the monitored process by comparing the online measured data with the control limits that are given in terms of the population standard deviation. Assume that $\left(x_{1}, x_{2}, \ldots, x_{n}\right)$ are individual observation collected from a monitored process. If the observations are Gaussian and uncorrelated, the control limits of the Shewhart chart can be computed as follows [12], [13]:

$$
U C L / L C L=\mu_{0} \pm Z_{1-\frac{\alpha}{2}} \sigma_{0},
$$

where $Z_{1-\frac{\alpha}{2}}$ denotes the $\left(1-\frac{\alpha}{2}\right)$-th quartile of the distribution $\mathcal{N}(0,1)$, which is also called the $\frac{\alpha}{2}$ critical value of the $\mathcal{N}(0,1)$ distribution. $\alpha$ is the probability of false alarm, $\mu_{0}$ and $\sigma_{0}$ represent the mean and the standard deviation of the healthy data set (i.e., data without falls), respectively. In the Equation 1, the critical value, $Z_{1-\frac{\alpha}{2}}$, which is termed the width of the control limits UCL and LCL, is usually specified in practice to be 3, which correspond to a false alarm rate of $0.27 \%$. An anomaly is signaled at time $t$ if $x_{t}<L C L$ or $x_{t}>U C L$. In summary, the Shewhart chart use only the data observed at specific time point to decide on the process performance at any one time point [12].

In this work, the detection algorithm is applied on accelerometer data. Specifically, we used the publicly available acceleration dataset from the University of Rzeszow's [8] in which the acceleration data were collected via an accelerometer X-IMU $(256 \mathrm{~Hz})$ devices. The acceleration data gathered via $\mathrm{x}$-IMU inertial sensor comprise the acceleration readings in triple-axis of $x-, y-$, and z-axis (i.e., a longitudinal acceleration, a lateral acceleration, a vertical acceleration). Here, the magnitude of acceleration, a, in three-dimensional space, which is the vector norm, is used as fall indicator and is defined as:

$$
a=\sqrt{a_{x}^{2}(t)+a_{y}^{2}(t)+a_{z}^{2}(t)},
$$

where $a_{x}(t), a_{y}(t)$ and $a_{z}(t)$ represent acceleration components according $x, y, z$ axes at the instant $t$. Example of acceleration change curves during a fall event are depicted in the top of Figure 1.

\section{FALL CLASSIFICATION}

As previously mentioned only detected sequences are concerned by the classification phase. During classification, only data from camera are taken into a count whereas data from sensors have already been used during detection stage. This classification phase is principally used for distinguishing between reel fall and like fall activities. In general, computer vision-based method includes different steps namely: image segmentation, feature extraction, and fall classification. In this section, each phase will be detailed where more explanations will be presented.
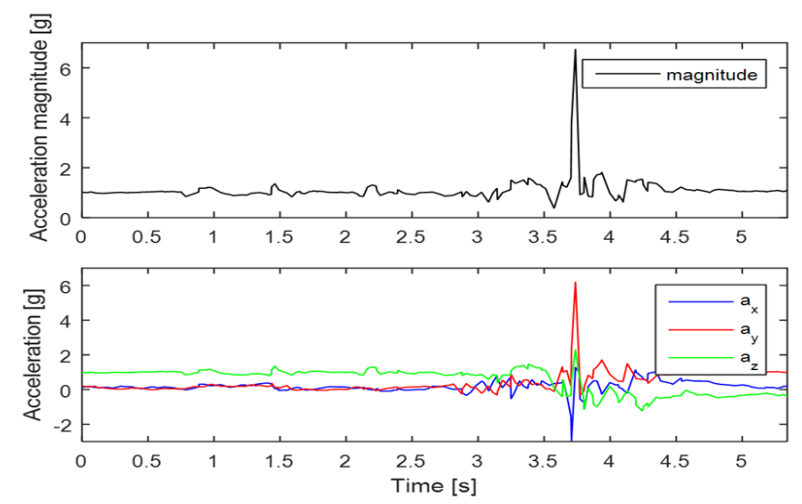

Figure 1. Acceleration (top row) and the tri-axial acceleration components observed while falling (bottom row).

\section{A. Segmentation and preprocessing}

The segmentation consists of extracting body's silhouette from the input image sequence. In this work, human body is typically discriminated using background subtraction technique. The background image is defined as reference to eliminate the unchanged pixels in the frame sequences [16]. This method is suitable here because it can manage multiple component models. However, some noise regions can be observed in the segmented image. To eliminate this noise, the morphological operator, which performs the erosion and dilation operator with $3 \times 3$ structuring elements, is applied.
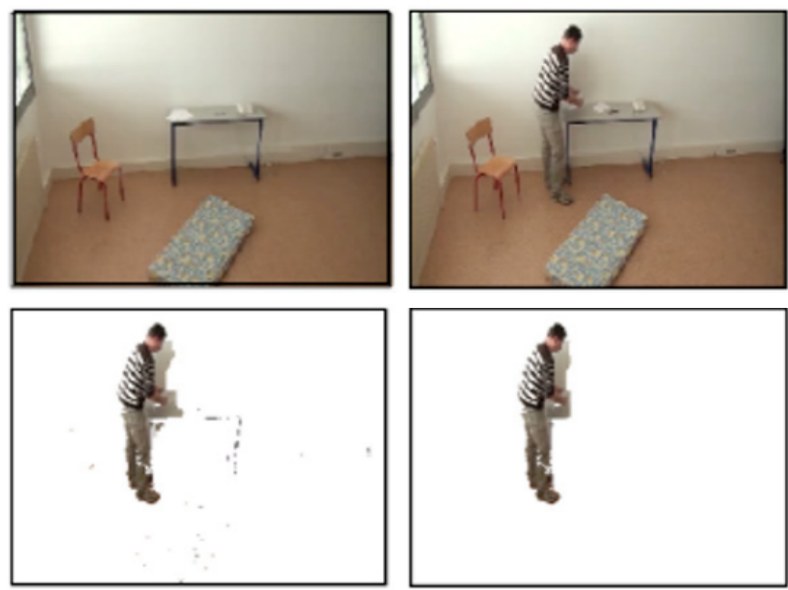

Figure 2. Results of the background subtraction algorithm.

Figure 2 illustrates an example of the background subtraction algorithm, where background and input frames are represented by the first colon, respectively, and the two images on the second colon, illustrate the respective results from background subtraction before and after applying morphological operators.

\section{B. Feature extraction}

Feature extraction is considered as crucial step in videobased fall classification, where extracted features have a di- 
8th International Conference on Modelling, Identification and Control (ICMIC-2016)

Algiers, Algeria- November 15-17, 2016

rect impact on the classification performances. The extracted features have to be invariant to image (i) translation when the position of human body changes in the image, and (ii) to scaling when the silhouette dimensions change as the distance between the monitored person and camera varies.
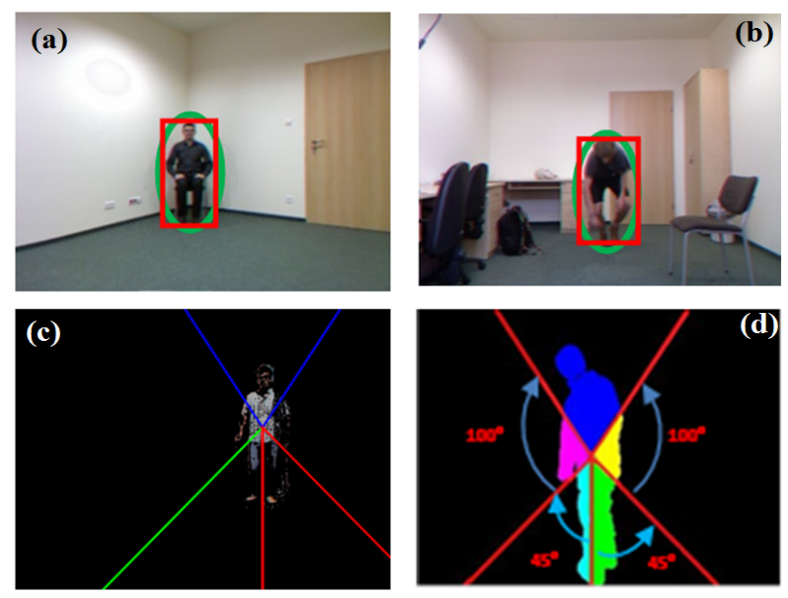

Figure 3. Features used in human posture description.

Recently, several works have focused on shape information to detect and classify falls. One can cite: the body's center of gravity [17], the bounding box and approximated ellipse of the silhouette. However, most of the proposed features cannot always distinguish among various body postures, especially when there is a high degree of similarity between activities (e.g., dimensions and orientations of the bounding boxes and approximated ellipses are nearly the same for both bending and sitting postures, as shown in Figure 3(a)-(b)). For this reason, we discard the idea to consider the body as a geometric shape. In this work, we base the extracted features on pixels constituting human body. More specifically, we use five partial occupancy areas of the body to detect and classify falls. These areas typically correspond to the action of body parts when in a standing posture, as shown in Figure 3(c)-(d). Finally, since frames of a video sequence are assimilated to an observation sequence, the set of ratios that are computed for each frame are then concatenated to form the whole feature vector corresponding to the video sequence. These areas are determined using a portioning centered on the body's gravity center $\left(x_{G}, y_{G}\right)$, which is simply the barycenter of the pixels.

$$
x_{G}=\frac{1}{N} \sum_{i=1}^{N} x_{i}, \quad y_{G}=\frac{1}{N} \sum_{i=1}^{N} y_{i},
$$

where $N$ represents the number of pixels representing the human body, and $x_{i}$ and $y_{i}$ denote the horizontal and vertical coordinates of pixels belonging to the human body, respectively. Finally, since frames of a video sequence are assimilated to an observation sequence, the set of ratios that are computed for each frame are then concatenated to form the whole feature vector corresponding to the video sequence.

\section{Neural network classifier}

Neural network classifier is a supervised algorithm, which can use multiple input, output and hidden layers with arbitrary number of neurons [15]. The most widely used neural classifier today is the multi-layer perception (MLP) network with back propagation (BP) learning algorithm. BP is used for the optimization of MLP. The classic architecture of an ANN classifier includes three layer types, namely: input layers, hidden layers, and output layers (see Figure 4). The input layers correspond generally to features to classify. The hidden layers are generally determined empirically and relatively to the expected classification accuracy. The output layer corresponds to the defined classes. Each class corresponds to a node in output layer. The output node value should provide the corresponding class for the input data, i.e. a high output value is expected on the correct class node and a low output value on all the rest (see Figure 4).

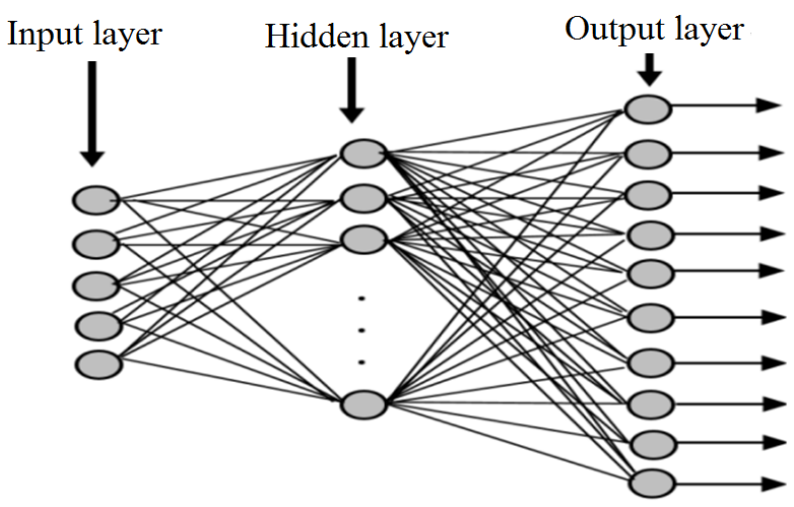

Figure 4. Multi-layer Neural Network Architecture.

During learning phase, a set of training input vectors is presented at the input layers by feature vectors and their corresponding desired output vectors. Initially random weights are assigned to the set of nodes. The neural network adjusts the weights attached to the connections according the difference between the network's output and the desired output for that input vector. More this difference is reduced more is better for classification. A single neuron in the network can be represented as follow:

$$
y_{i}=f_{i} \sum w_{i j} \times x_{i}
$$

where, $x_{i}$ is data input to neural network, $w_{i j}$ represents weights between $i^{\text {th }}$ neuron of previous layer and $j^{\text {th }}$ neuron of the current layer and $f_{j}$ represents the activation function. Various activation functions exist in the literature, one can cite: linear, sigmoid, hyperbolic tangent. It is worth noting that neural network classifier is exploited in a numerous fall detection applications [18]. However, neural network classification presents some limitations. Neural Networks use an empirical risk minimization and non-structural risk minimization. In addition ANNs are more prone to over fitting problem. 
8th International Conference on Modelling, Identification and Control (ICMIC-2016)

Algiers, Algeria- November 15-17, 2016

\section{EXPERIMENTAL RESULTS}

In this section, we evaluate the abilities of the Shewhartbased fall detector with the Neural Network classifier to detect and classify falls.

\section{A. Detection results}

Two examples are presented here to illustrate the capacity of the shewhart-based fall detector to detect a fall event. In the first example, the testing data used to test the performance of the Shewhart chart contain a fall from walking or standing position. The results of the Shewhart chart and its corresponding accelerometric data are illustrated in Figure 5, and clearly show that this chart detected the fall without false alarms.

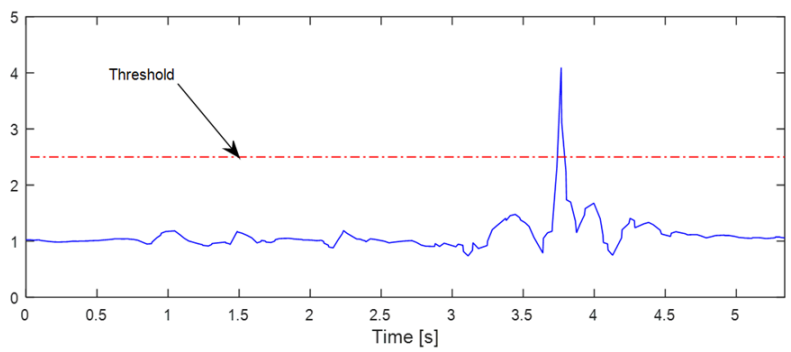

Figure 5. Monitoring results of Shewhart chart in the case of falling from stating position. The horizontal dashed line denotes the control limit.

In the second case example, the testing data contain lying down on the floor or false falls. The results using the Shewhart chart (shown in Figure 6) show that it could successfully detect this false fall. We note that the Shewhart chart cannot

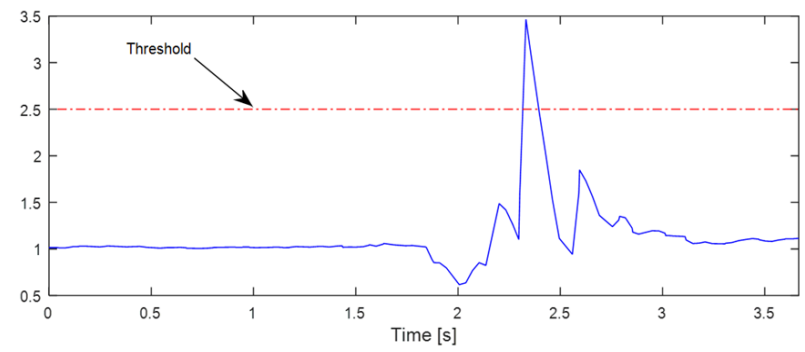

Figure 6. Monitoring results of Shewhart chart in the case of lying down on the floor. The horizontal dashed line denotes the control limit.

distinguish real falls from certain fall-like actions such as lying down. To deal with this problem a classification module should be added after fall detection.

\section{B. Classification results}

To assess the detection ability of the developed Shewhartneural network fall detection strategy, we performed experiments on the publicly existing fall detection databases: the UR fall detection dataset (URFD) [19]. URFD comprises 70 sequences of several actions performed in different ways. Falls and activities of daily living (ADL) used in this work are recorded with an RGB camera. The URFD comprises 30 images per sequence for both classes: falls and typical ADLs. All sequences are recorded with color cameras and synchronized with their corresponding accelerometer data. We evaluated the Shewhart-based neural network classifier and compared it with neural network classifier. We used a 3-fold cross-validation to evaluate the classifiers. Table I compares the proposed fall detection and classification strategy of the Shewhart-neural network with that using neural network alone with no detection phase.

Table I

CLASSIFICATION PERFORMANCE COMPARISON

\begin{tabular}{|l|c|c|c|c|c|c|c|}
\hline Classifier & Accuracy & Se & Sp & Precession & Recall & F-measure & AUC \\
\hline NN & 95.16 & 1 & 0.91 & 0.903 & 1 & 0.95 & 0.95 \\
\hline Shewhart-NN & 96.67 & 1 & 0.934 & 0.931 & 1 & 0.96 & 0.96 \\
\hline
\end{tabular}

The results shown in Table I demonstrate that the integrated Shewhart-neural network strategy is more accurate at detecting falls than neural network classifier alone, indicating that combining the detection phase with a classifier allows us to distinguish between daily activities and falls, reducing the space of training and testing data used as input for classification. Furthermore, because the neural network classification is only applied to the data corresponding to detected cases, a reduced number of video sequences need to be classified, which makes processing much faster than when all data must be classified .

\section{CONClusion}

In this work, information from acceleration sensors and camera are both used to design reliable fall detection strategy. The results show that combining Shewhart chart with neural network classifier was provide good separation of true falls from fall-like events. We utilize Shewhart chart to identify features corresponding to falls that are pertinent for fall classification phase. Furthermore, the Shewhart chart plays an important role in reducing the size of the features used as input data to neural network for classification, which significantly reduces the computational burden and achieves reasonable accuracy. Results demonstrate the superior classification capacity of Shewhart-based neural network algorithm compared with neural network alone.

\section{ACKNOWLEDGEMENT}

This publication is based upon work supported by King Abdullah University of Science and Technology (KAUST) Office of Sponsored Research (OSR) under Award No: OSR2015-CRG4-2582.

\section{REFERENCES}

[1] W. H. O. Ageing and L. C. Unit, WHO global report on falls prevention in older age. World Health Organization, 2008.

[2] C. Todd and D. Skelton, What are the Main Risk Factors for Falls Amongst Older People and what are the Most Effective Interventions to Prevent These Falls?. World Health Organization, 2004.

[3] M. Yu, A. Rhuma, S. Naqvi, L. Wang, and J. Chambers, "A posture recognition-based fall detection system for monitoring an elderly person in a smart home environment," IEEE transactions on information technology in biomedicine, vol. 16, no. 6, pp. 1274-1286, 2012.

[4] T. Soriano, L. DeCherrie, and D. Thomas, "Falls in the communitydwelling older adult: a review for primary-care providers," Clinical interventions in aging, vol. 2, no. 4, p. 545, 2007. 
8th International Conference on Modelling, Identification and Control (ICMIC-2016)

Algiers, Algeria- November 15-17, 2016

[5] Y. Delahoz and M. Labrador, "Survey on fall detection and fall prevention using wearable and external sensors," Sensors, vol. 14, no. 10, pp. 19806-19842, 2014.

[6] P. Zweifel, S. Felder, and M. Meiers, "Ageing of population and health care expenditure: a red herring?" Health economics, vol. 8, no. 6, pp. 485-496, 1999.

[7] M. Mubashir, L. Shao, and L. Seed, "A survey on fall detection: Principles and approaches," Neurocomputing, vol. 100, pp. 144-152, 2013.

[8] B. Kwolek and M. Kepski, "Improving fall detection by the use of depth sensor and accelerometer," Neurocomputing, vol. 168, pp. 637645, 2015.

[9] C. Rougier, J. Meunier, A. St-Arnaud, and J. Rousseau, "Robust video surveillance for fall detection based on human shape deformation," IEEE Transactions on Circuits and Systems for Video Technology, vol. 21, no. 5, pp. 611-622, 2011

[10] P. Veltink, H. Bussmann, W. Vries, W. Martens, and R. V. Lummel, "Detection of static and dynamic activities using uniaxial accelerometers," IEEE Transactions on Rehabilitation Engineering, vol. 4, no. 4, pp. 375-385, 1996.

[11] F. Harrou, N. Zerrouki, Y.Sun, and A.Houacine, "A simple strategy for fall events detection," in IEEE International Conference on Industrial Informatics, INDIN 2016, 2016.

[12] D. Montgomery, Introduction to statistical quality control. John Wiley \& Sons, 2007.

[13] F. Kadri, F. Harrou, S. Chaabane, Y. Sun, and C. Tahon, "Seasonal ARMA-based SPC charts for anomaly detection: Application to emergency department systems," Neurocomputing, vol. 173, pp. 2102-2114, 2016.

[14] F. Harrou, Y. Sun, and S. Khadraoui, "Amalgamation of anomalydetection indices for enhanced process monitoring," Journal of Loss Prevention in the Process Industries, vol. 40, pp. 365-377, 2016.

[15] J. Hertz, A. Krogh, and R. Palmer, Introduction to the theory of neural computation. Basic Books, 1991, vol. 1.

[16] N. Zerrouki, F. Harrou, Y. Sun, and A. Houacine, "A data-driven monitoring technique for enhanced fall events detection," IFACPapersOnLine, vol. 49, no. 5, pp. 333-338, 2016.

[17] C.-L. Liu, C.-H. Lee, and P.-M. Lin, "A fall detection system using knearest neighbor classifier," Expert systems with applications, vol. 37, no. 10 , pp. $7174-7181,2010$.

[18] H. Foroughi, B. Aski, and H. Pourreza, "Intelligent video surveillance for monitoring fall detection of elderly in home environments," in 11th International Conference onComputer and Information Technology, ICCIT 2008. IEEE, 2008, pp. 219-224.

[19] B. Kwolek and M. Kepski, "Human fall detection on embedded platform using depth maps and wireless accelerometer," Computer methods and programs in biomedicine, vol. 117, no. 3, pp. 489-501, 2014. 